\title{
Striking a balance in an antibody network: A roadmap for HIV-1 vaccines
}

\author{
Tysheena P. Charles ${ }^{1,2}$ and Cynthia A. Derdeyn ${ }^{1,2,3}$ \\ ${ }^{1}$ Emory Vaccine Center, ${ }^{2}$ Yerkes National Primate Center, and ${ }^{3}$ Department of Pathology and Laboratory Medicine, Emory University, Atlanta, Georgia, USA.
}

\begin{abstract}
With almost 2 million new HIV-1 infections in 2018, a highly effective vaccine is imperative. Vaccine-elicited HIV-1 antibodies contribute to protection through multiple nonneutralizing activities, but the exact mechanisms remain unknown. In this issue of the $J C I$, Neidich and associates sought to determine how antibodies contributed to reducing the risk of HIV-1 acquisition in a phase Ilb preventative vaccine efficacy trial, HVTN 505. Their studies revealed that antibody-dependent cellular phagocytosis (ADCP) and Fc $\gamma \mathrm{R}$ Ila binding were strongly associated with reduced HIV-1 risk; however, HIV-1 envelope-specific IgC3, IgA; and host FcyRIla genotype also influenced risk. This study highlights the intricate interactions between antibodies and innate immune functions in humans.
\end{abstract}

\section{Antibody Fc interactions correlated with reduced HIV-1 acquisition}

HIV-1 remains the leading cause of death worldwide, with 38 million people living with HIV/AIDS and 770,000 HIV-infected people dying in 2018 (1). Despite drug- and behavior-based interventions, new cases still occur at an alarming rate in many regions of the world. A safe and effective vaccine could significantly decrease new HIV infections, save millions of lives, and reduce the burden of AIDS on a global scale. An effective vaccine will need to induce robust and long-lasting humoral and cellular immune responses that can overcome genetically diverse HIV-1 variants with complex immune evasion strategies. Recent studies of immune correlates in vaccinated human volunteers have revealed complicated profiles of $\mathrm{Ig}$ antibody class, subtype, and effector functions, suggesting that fine-tuning antibody profiles could provide better protection against HIV-1 infection (2-5). Neidich et al. investigated how nonneutralizing antibody responses contributed to reduced acquisition risk in a phase IIb efficacy trial, HVTN 505, which was conducted at 21 sites within the US (6). The trial administered DNA and recombinant adenovirus type 5 (rAd5) immunizations to 2,500 men and transgendered persons who have sex with men and were at risk for HIV-1 infection (7). The vaccine was designed to elicit antibodies against envelope (Env) proteins representing three major HIV-1 clades as well as multifunctional $\mathrm{CD} 4^{+}$and $\mathrm{CD}^{+} \mathrm{T}$ cells (7). There was no effect of the vaccine on acquisition of HIV-1 infection. However, the vaccine left a footprint of immune pressure on the Env protein of breakthrough variants (8). Further, it elicited a high rate of antibody-dependent cellular phagocytosis (ADCP) with a broad dynamic range (8). To identify immune risk correlates, the authors used a case-control strategy in which they compared immune measures between 125 uninfected and 25 infected vaccine recipients, sampled at 4 weeks after the final rAd5 immunization (6).

Neutralizing-antibody activity has been associated with vaccine-mediated protection against several pathogens; how-

Related Article: p. 4838

Conflict of interest: The authors have declared that no conflict of interest exists.

Copyright: @ 2019, American Society for Clinical Investigation.

Reference information: J Clin Invest. 2019;129(11):4580-4582. https://doi.org/10.1172/JCl132535.

ever, antibodies exhibiting a much more diverse portfolio of antiviral activities may better reduce susceptibility to HIV-1. Capitalizing on antibody diversity might prove advantageous if the antibodies were abundant, could bind to different virion forms and Env proteins, could interact with multiple $\mathrm{Fc} \gamma$ receptors ( $\mathrm{Fc} \gamma \mathrm{Rs}$ ), and required low mutation without unusual adaptations (9). Antibody binding to Fc $\gamma$ Rs expressed on various effector cells provides a powerful bridge between the innate and adaptive arms of the immune system. This interaction can lead to such effector functions as antibody-dependent cell-mediated cytotoxicity (ADCC) of infected cells or ADCP of opsonized viral particles, which is typically mediated by IgG3 antibodies engaged with Fc $\gamma$ RIIa $(10,11)$.

To gain insight into whether the ADCP generated by HVTN 505 was a correlate of reduced risk, Neidich et al. evaluated this activity in vitro using a consensus HIV-1 Env protein coupled to fluorescent beads, which are taken up by a monocytic cell line upon antibody binding to Fc $\gamma$ RIIa (12). This approach detected Env-specific, IgGmediated ADCP in $88 \%$ of the vaccinees, and the median ADCP score was twice as high in uninfected individuals compared with infected individuals (6). The authors also measured in vitro binding of tetrameric Fc $\gamma$ RIIa to Env-specific IgG. ADCP and Fc $\gamma$ RIIa binding both significantly correlated with reduced risk of HIV-1 acquisition, which held when controlling for potentially confounding factors such as age, race, behavior risk, and body mass index (6). Further analyses demonstrated high IgG3 response rates in HVTN 505 vaccinees, and IgG3 binding to a panel of HIV-1 Env proteins was also significantly correlated with reduced risk of HIV-1 acquisition (6). These results support a scenario in which high IgG3 responses against Env facilitated Fc $\gamma$ RIIa engagement and ADCP, which contributed to reducing the infection risk. While these results provide new insight into the protective potential of a subset of 
nonneutralizing IgG antibodies, further studies could reveal a clearer understanding of the mechanisms. Antibody binding to FcyRIIa could have resulted in phagocytosis of opsonized viral particles, but could also have induced signaling by the effector cells, providing indirect antiviral activity. It will also be critical to understand why vaccine-elicited ADCP did not provide a higher level of protection against infection. The authors postulated that the short IgG3 halflife in serum limited protection, so maximizing antiviral activity will likely require developing approaches to increase anti-Env IgG3 magnitude and durability. Further experiments should also test ADCP using viral variants that represent circulating strains and breakthrough variants from this cohort. Ultimately, characterizing the specificity of antibodies that mediate ADCP will guide the design of vaccine immunogens that can present effective targets.

\section{Other immune and host factors modulated HIV-1 infection risk}

It is becoming more evident that Env-specific circulating IgA elicited by vaccination could indirectly modulate IgG antibody function. In the HIV-1 vaccine efficacy trial RV144, Env-specific plasma IgA directly associated with HIV-1 infection risk, possibly by interfering with protective IgG antibodies that could mediate ADCC and other antiviral activities $(5,13)$. When the authors investigated Env-specific IgA in HVTN 505 vaccinees, the vaccine, again, exhibited a negative impact on risk. High Env-specific IgA levels negatively modified the association between ADCP and reduced risk of acquisition (6). In other words, when Env-specific IgA levels were low or undetectable, the potentially protective effects of ADCP and antibody binding to Fc $\gamma$ RIIa were even stronger. It is worth noting that IgA exists as two isotypes in humans and is found in multiple specialized forms in blood and mucosal tissue compartments $(14,15)$. Like IgG antibodies, serum IgA can interact with $\mathrm{Fc} \gamma \mathrm{R}$, mediate antiviral activities, and is also thought to play a key role in balancing inhibitory and activating pathways that influence IgG-mediated functions (14). Thus, elevated serum IgA may serve as a marker of a dysregulated humoral immune response. It will be interesting to more fully characterize the properties of vaccine-induced serum and mucosal IgA antibodies and determine whether they do, in fact, interfere with the capacity of IgG to mediate ADCP, directly or indirectly. Future studies could entertain the possibility of developing vaccine regimens that generate balanced amounts of IgA and IgG.

Given the strong association between Fc $\gamma$ RIIa binding and reduced risk of infection, Neidich and colleagues considered the genetics of the vaccinated individuals by investigating whether polymorphisms in the Fc $\gamma$ RIIa gene modified the correlation of antibody functions with risk of infection. They found that the presence of a certain genotype strengthened the association of ADCP with reduced risk of acquisition, and this association remained highly significant when adjusted for cellular responses (6). These results highlight how host genetic variation can impact the underlying Fc-mediated antibody functions and influence HIV-1 susceptibility.

Although antibodies repeatedly correlate with reduced risk in vaccinated humans, it is logical to expect that robust cellular immune responses could also contribute to an effective HIV-1 vaccine by containing initial viral spread or controlling breakthrough HIV-1 replication. Previous studies have provided evidence that cell-mediated immune responses are not protective on their own, but do exert selective pressure on breakthrough virus (16). Neidich et al. investigated whether there were any potential synergistic effects between cellular and humoral responses in reducing acquisition in HVTN 505. They used systems analysis and machine-learning approaches to search for evidence that polyfunctional $\mathrm{CD} 4^{+}$and $\mathrm{CD} 8^{+} \mathrm{T}$ cells also contributed to reducing infection risk. When they tested all measured immune responses and baseline clinical and demographic factors for prediction of risk, they found that antibody and $\mathrm{T}$ cell variables together gave the highest predictive power, indicating that multiple immune components likely contributed to defining HIV-1 risk in HVTN 505. However, future studies should expand on how to enhance the immune responses that track with reduced acquisition, both antibody and $\mathrm{T}$ cell mediated, to the point that together they can provide a high level of protection against acquisition.

Lastly, the real-world complexity of a vaccine clinical trial such as HVTN 505 should not be underappreciated. HVTN 505 involved multiple sites, a wide age range, racial and ethnic diversity, variation in risk behaviors, sexually transmitted infections, use of pre- and postexposure prophylaxis, and drug and alcohol use (7). Even though Neidich et al. controlled for potentially confounding factors, it is remarkable that such strong antibody correlates of HIV-1 risk were identified. Although the effect failed to prevent infection, the mechanisms could have a direct impact on improving clinical vaccines.

\section{Conclusions and future directions}

It is apparent that vaccines can elicit antibodies that mediate functions such as $\mathrm{ADCC}$ and $\mathrm{ADCP}$, as measured in vitro. These vaccine-related responses continue to emerge as immune correlates of reduced acquisition, suggesting that we need to investigate the intricate network of IgG and IgA antibodies circulating in serum and located at mucosal surfaces, and how different antibody types synergize or compete to converge on effector functions to modulate risk of infection. The work by Neidich et al. suggests that a vaccine should aim to elicit highly functional and carefully orchestrated humoral immune responses that strike a balance between functions, classes, and subtypes, instead of relying on a single type of antibody or function to provide comprehensive protection. Magnitude and durability of functional antibody responses against Env remain as challenges to overcome. The study here also reminds us that $\mathrm{Fc} \gamma \mathrm{R}$ host genetics and immune repertoire modulate immune activity and warrant further investigation. Future experiments should utilize different Env proteins, virions, or stabilized trimers, breakthrough and/or circulating viral variants, and autologous primary effector cells, and develop additional approaches to define mechanisms beyond the immune measure in serum. In the absence of a highly efficacious HIV-1 vaccine, new information about how antibodies could reduce the risk of infection provides viable avenues of investigation.

\section{Acknowledgments}

We would like to thank Samantha Burton, Salar Khan, and Eric Hunter for their helpful comments. This work was supported 
by R01 AI128837 and UM1 AI124436 from the National Institute of Allergy and Infectious Diseases, NIH.

Address correspondence to: Cynthia A. Derdeyn, Emory University, Yerkes National Primate Research Center, 954 Gatewood Rd., Atlanta, Georgia 30329, USA. Phone: 404.727.8594; Email: cderdey@ emory.edu.

1. UNAIDS. Global HIV \& AIDS statistics - 2019 fact sheet. http://www.unaids.org/en/resources/ fact-sheet. Accessed September 17, 2019.

2. Tomaras GD, Plotkin SA. Complex immune correlates of protection in HIV-1 vaccine efficacy trials. Immunol Rev. 2017;275(1):245-261.

3. Bournazos S, Ravetch JV. Fc $\gamma$ receptor function and the design of vaccination strategies. Immunity. 2017;47(2):224-233.
4. Gao Y, McKay PF, Mann JFS. Advances in HIV-1 vaccine development. Viruses. 2018;10(4):E167.

5 . Haynes BF, et al. Immune-correlates analysis of an HIV-1 vaccine efficacy trial. N Engl J Med. 2012;366(14):1275-1286.

6. Neidich SD, et al. Antibody Fc effector functions and IgG3 associate with decreased HIV-1 risk. JClin Invest. 2019;129(11):4838-4849.

7. Hammer SM, et al. Efficacy trial of a DNA/ rAd5 HIV-1 preventive vaccine. $N$ Engl J Med. 2013;369(22):2083-2092.

8. deCamp AC, et al. Sieve analysis of breakthrough HIV-1 sequences in HVTN 505 identifies vaccine pressure targeting the CD4 binding site of Env-gp120. PLoS ONE. 2017;12(11):e0185959.

9. Nimmerjahn F, Ravetch JV. Antibody-mediated modulation of immune responses. Immunol Rev. 2010;236:265-275.

10. Watzl C, Long EO. Signal transduction during activation and inhibition of natural killer cells. Curr Protoc Immunol. 2010; Chapter 11:Unit 11.9B.

11. Zhang Y, Hoppe AD, Swanson JA. Coordination of Fc receptor signaling regulates cellular commitment to phagocytosis. Proc Natl Acad Sci U S A. 2010;107(45):19332-19337.

12. Ackerman ME, et al. A robust, high-throughput assay to determine the phagocytic activity of clinical antibody samples. J Immunol Methods. 2011;366(1-2):8-19.

13. Tomaras GD, et al. Vaccine-induced plasma IgA specific for the $\mathrm{C} 1$ region of the HIV-1 envelope blocks binding and effector function of IgG. Proc Natl Acad Sci U S A. 2013;110(22):9019-9024.

14. Leong KW, Ding JL. The unexplored roles of human serum IgA. DNA Cell Biol. 2014;33(12):823-829.

15. Lopez E, Shattock RJ, Kent SJ, Chung AW. The multifaceted nature of immunoglobulin A and its complex role in HIV. AIDS Res Hum Retroviruses. 2018;34(9):727-738.

16. Iaccino E, Schiavone M, Fiume G, Quinto I, Scala G. The aftermath of the Merck's HIV vaccine trial. Retrovirology. 2008;5:56. 